\title{
Supporting Information: Wet-stamped precipitant gradients control the growth of protein microcrystals in an array of nanoliter wells.
}

\author{
Goher Mahmud, Kyle J.M. Bishop, Yuriy Chegel, Stoyan K. Smoukov, and \\ Bartosz Grzybowski* \\ Department of Chemical and Biological Engineering and \\ Department of Chemistry \\ Northwestern University, 2145 Sheridan Road, Evanston, IL 60208
}

\section{Materials and Methods}

(a) Microwell array. A $2 \mathrm{~cm} \times 2 \mathrm{~cm} \times 1 \mathrm{~cm}$ rectangular slab of poly (dimethyl siloxane) (PDMS, SYLGARD 184) having an array of $100 \mu \mathrm{m}$ x $100 \mu \mathrm{m}$ x $150 \mu \mathrm{m}$ square wells (spaced by $100 \mu \mathrm{m})$ embossed on its surface was fabricated by molding against a photolithographically patterned SU8 master (S1). Wells were filled with hot $\left(100^{\circ} \mathrm{C}\right)$, degassed $1 \% \mathrm{w} / \mathrm{v}$ agarose (High gel strength, OmniPur, LOT 2090), cooled to gel, and soaked overnight in a buffer solution (Acetate, $\mathrm{pH} 4.65$, Riedel-de-Hen, LOT 36050) of $60 \mathrm{mg} / \mathrm{mL}$ lysozyme (from chicken egg white, Sigma, LOT L6876).

(b) Precipitant reservoir (“stamp”). Hot, degassed, agarose (10\% w/v) was cooled to gel, cut into $\sim 1 \mathrm{~cm} \times 1 \mathrm{~cm} \times 2 \mathrm{~cm}$ blocks, and soaked overnight in a $15 \% \mathrm{w} / \mathrm{v}$ aqueous solution of $\mathrm{NaCl}$ (Sigma-Aldrich, LOT 310-166).

(c) Transport layer. Hot, degassed, agarose $(5 \% \mathrm{w} / \mathrm{v})$ was sandwiched between two glass plates separated by $400 \mu \mathrm{m}$ spacers, cooled to gel, and cut into $2 \mathrm{~cm} \times 2 \mathrm{~cm}$ sheets.

(d) Dialysis membrane. Dialysis tubing cellulose membrane (D9777, Sigma-Aldrich) was cut into $2 \mathrm{~cm} \times 2 \mathrm{~cm}$ sheets and soaked in buffer solution.

(e) Assembly procedure. The membrane and the transport layers were carefully (to avoid trapping air bubbles) placed onto the PDMS substrate presenting protein-containing microwells. The agarose stamp containing $\mathrm{NaCl}$ was then placed near an edge of the PDMS support, and the entire assembly was immersed in paraffin oil (Paraffin, J.T. Baker, LOT C11H17) to prevent any evaporation and gel drying during the crystallization process. 
2. Image acquisition was performed on an optical microscope (Nikon OPTIPHOT-2 microscope interfaced with SPOT software). Images of the growing crystals were taken in $\sim 1 \mathrm{hr}$ time intervals for at least 50 hours.

3. Figure S1.Additional images of microcrystals grown at $50 \mathrm{hrs}$ in rows located at $\mathrm{X} 1 \sim 4 \mathrm{~mm}$, $\mathrm{X} 2 \sim 6 \mathrm{~mm}, \mathrm{X} 3 \sim 16 \mathrm{~mm}, \mathrm{X} 4 \sim 18 \mathrm{~mm}$ from the $\mathrm{NaCl}$ source.

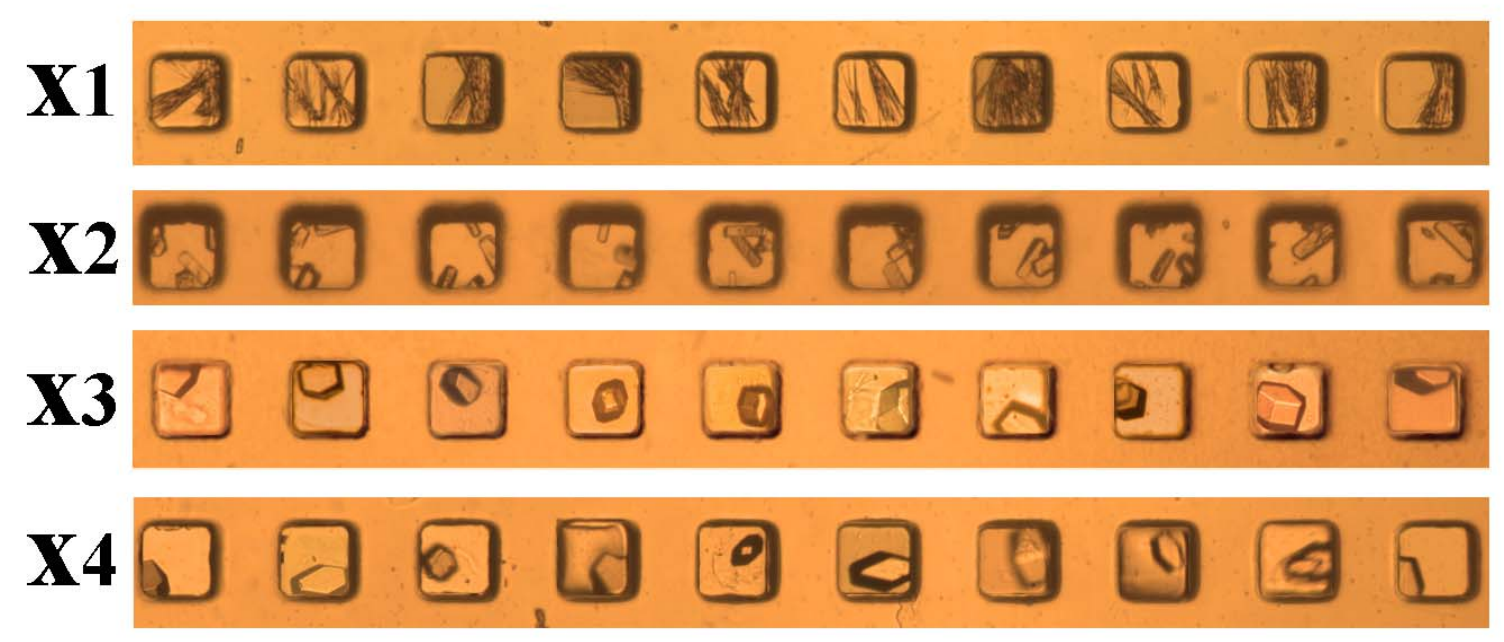

\section{Reaction-diffusion modeling.}

(a) Precipitant Concentration. Time-dependent salt concentration profiles were calculated from one-dimensional (1D) diffusion equation, $\partial C_{S} / \partial t=D \partial^{2} C_{S} / \partial x^{2}$, over $0 \leq x \leq L$ domain, where $x=0$ corresponds to the edge of the stamp and $x=\mathrm{L}$ to the far end of the gel substrate (cf. Fig. 1). Here, $C_{S}(x, t)$ is the salt concentration at position $x$ and time $t$, and $D$ is the diffusion coefficient ( $D \approx 5 \times 10^{-6} \mathrm{~cm}^{2} / \mathrm{s}$ in $10 \% \mathrm{w} / \mathrm{v}$ agarose gel (S2)). Initially, the salt concentration is zero in the gel substrate, $C_{S}(x, 0)=0$. At $t=0$, the stamp is applied and maintains a constant salt concentration, $C_{S}^{0}$, at $x=0$ (i.e., $\left.C(0, t)=C_{S}^{0}\right)$; the far boundary is impermeable to diffusion such that $\partial C(L, t) / \partial x=0$. With these initial and boundary conditions, the solution to the diffusion equation is:

$$
C(x, t)=C_{S}^{0}\left[1-\frac{2}{\pi} \sum_{n=1}^{\infty} \frac{1}{(n-1 / 2)} \sin \left(\frac{(n-1 / 2) \pi x}{L}\right) \exp \left(-\frac{(n-1 / 2)^{2} \pi^{2} D t}{L^{2}}\right)\right]
$$

This solution neglects any effects due to the wells, which are small (100 $\mu \mathrm{m}$ deep) compared to the thickness of the gel layer above them $(400 \mu \mathrm{m})$. Furthermore, it is assumed that the concentration within the wells is approximately uniform and equal to $C_{S}(x, t)$, where $x$ is the distance from the center of the well to the stamp. This assumption is justified considering that the 
characteristic diffusion time for achieving a uniform concentration within a well, $\tau_{D}=(100 \mu \mathrm{m})^{2} /\left(10^{-6} \mathrm{~cm}^{2} / \mathrm{s}\right)=100 \mathrm{~s}$, is short compared to the time scale of the experiments (i.e., $\tau_{D} \ll \tau_{E x p} \approx 50 \mathrm{hrs}$ ). With the above assumptions, the above equation provides the time evolution of the salt concentration in each well (cf. Fig. 2a).

(b) Nucleation and Growth Model. To model crystal growth, we developed a model based on the nucleation and growth mechanism, which accounts for the changing precipitant concentration, stochastic nucleation of crystal seeds, and the growth rates of the two distinct crystal faces. Here, the precipitant $(\mathrm{NaCl})$ concentration was given by the diffusion equation solution detailed in the previous section. It was assumed that the salt concentration depended only on time and the position of the well - i.e., not on the processes of nucleation and crystal growth occurring within the well.

Crystal nucleation was modeled using an empirical relation based on classical nucleation theory in which the nucleation rate is given as $J=A C_{P} \exp \left\{-B /\left[\ln \left(C_{P} / C_{P}^{*}\right)\right]\right\}$, where $C_{P}$ is the protein concentration, $C_{P}^{*}$ is the equilibrium protein solubility, and $A$ and $B$ are empirically determined parameters (here, $A=60,000$ crystals $\mathrm{mg}^{-1} \mathrm{~min}^{-1}$ and $B=85$ for lysozyme) (S3). Importantly, the protein solubility depends on the precipitant concentration as approximated by $C_{P}^{*}=(0.087 \% \mathrm{w} / \mathrm{v}) \exp \left[-C_{S} /(0.018 \% \mathrm{w} / \mathrm{v})\right](S 4)$.

Once nucleated, the crystals "seeds" grow at a rate that depends on both the protein and the precipitant concentrations. Furthermore, since lysozyme crystals have two distinct crystal faces (110 and 101), it is necessary to consider the growth rates of each face in order to describe/predict the growth of anisotropic crystals. These growth rates for lysozyme have been carefully measured in previous studies (S5). Here, the growth rates, $G_{110}$ and $G_{101}$, are defined as the velocity of a crystal face in the direction normal to that face.

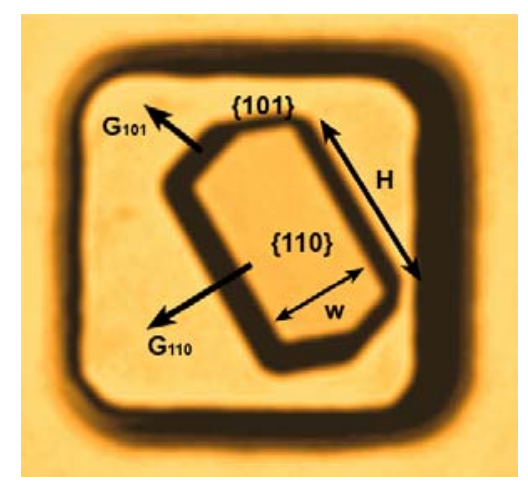

Figure S2. The faces and growth directions of a tetragonal lysozyme crystal. 
The dependence of the growth rate $G$ on the protein concentration was approximated by $G=k C_{P} \ln \left(C_{P} / C_{P}^{*}\right)^{n}$, where $k$ and $n$ are fitting parameters, both of which depend on the precipitant concentration (S5). For example, for $5 \% \mathrm{w} / \mathrm{v} \mathrm{NaCl}$, the "best-fit" parameters to the data of Durbin and Feher (S5) are $k_{110}=5 \times 10^{-3}, n_{110}=3.2, k_{101}=7 \times 10^{-3}$, and $n_{101}=2.1$; however, for $3.5 \% \mathrm{w} / \mathrm{v} \mathrm{NaCl}$, the "best-fit" parameters are $k_{110}=8 \times 10^{-5}, n_{110}=6.2, k_{101}=7 \times 10^{-4}$, and $n_{101}=$ 3.9. As the precipitant $(\mathrm{NaCl})$ concentration in our experiments varied considerably during the crystallization process (i.e., from 0 to $15 \% \mathrm{w} / \mathrm{v}$ ), we estimated $k$ and $n$ for the two faces via linear interpolation using the known "best-fit" values given above.

The above processes of nucleation and growth were coupled through the concentration of protein in the wells. To describe the evolution of this concentration, we considered the depletion of protein from each well as the result of crystal growth. For simplicity, we approximated the geometry of the crystals as rectangular with dimensions $W \times W \times H$ (corresponding to the schematic in Figure S1). With this simplification, the rate of protein depletion became:

$$
\frac{d C_{P}}{d t}=-\frac{1}{v_{P} V_{\text {well }}}\left(\sum_{i=1}^{N} W_{i}^{2} \frac{d H_{i}}{d t}+2 H_{i} W_{i} \frac{d W_{i}}{d t}\right)
$$

where $v_{P}$ is the molar volume of the protein crystal, $\mathrm{V}_{\text {well }}$ is the volume of the well, and the sum is carried out over all $N$ crystals in the well. Furthermore, the rates $d H / d t$ and $d W / d t$ are directly related to the growth rates $G_{110}$ and $G_{101}$ via the crystal geometry: $d W / d t=2 G_{110}$ and $d H / d t \approx 2.2 G_{101}(S 5)$.

In sum, the above equations amount to $2+2 N$ coupled, first order differential equations needed to solve for the number of crystals $N$ in the well, the dimensions $H_{i}$ and $W_{i}$ of each crystal, and the protein concentration $C_{P}$ :

Nucleation:

$$
J=A C_{P} \exp \left[\frac{-B}{\ln \left(C_{P} / C_{P}^{*}\right)}\right]
$$

Crystal Growth: $\quad \frac{d W_{i}}{d t}=2 k_{110} C_{p} \ln \left(C_{P} / C_{P}^{*}\right)^{n_{110}}$ and $\frac{d H_{i}}{d t}=2.2 k_{101} C_{p} \ln \left(C_{P} / C_{P}^{*}\right)^{n_{101}}$ for $i$ $=1 \ldots N$

Protein Depletion: $\quad \frac{d C_{P}}{d t}=-\frac{1}{v_{P} V_{\text {well }}}\left(\sum_{i=1}^{N} W_{i}^{2} \frac{d H_{i}}{d t}+2 H_{i} W_{i} \frac{d W_{i}}{d t}\right)$

In the above system of equations, $C_{P}^{*}=(0.087 \% \mathrm{w} / \mathrm{v}) \exp \left[-C_{S} /(0.018 \% \mathrm{w} / \mathrm{v})\right]$ and the salt concentration $C_{S}$ is given by the solution of diffusion equation for position $x$ and time $t$. 
(c) Numerical Methods. To solve these equations, we employed a fixed time step integration scheme for the nucleation term and an adaptive step-size implementation of the $4^{\text {th }}$ order RungeKutta method for the crystal growth and protein depletion terms. Because the number of crystals may take only integer values, nucleation was treated stochastically at each time step $\Delta t$. Briefly, at time $t$ a new crystal seed is created with probability $p_{\text {nuc }}=J V_{\text {well }} \Delta t$ and the equations are then integrated from time $t$ to $t+\Delta t$ via the $4^{\text {th }}$ order Runge-Kutta scheme, during which the existing crystals grow and deplete their protein reservoir. At time $t+\Delta t$, another nucleation is attempted and the procedure is repeated. Given the stochastic nature of the model, multiple realizations/runs needed to be performed (tens to hundreds) to obtain reliable statistics for each well position $X_{\text {well }}$.

\section{References}

S1. Campbell, C.J.; Fialkowski, M.; Klajn, R.; Bensemann, I.T.; Grzybowski, B.A. Adv. Mater. 2004, 16, 1912.

S2. $\quad$ K.J.M. Bishop, B.A. Grzybowski. Phys. Rev. Lett. 2006, 97, 12.

S3. $\quad$ Bhamidi, V.; Varanasi, S.; Schall, C.A. Crys.Growth \& Des. 2002, 2, 5.

S4. Durbin, S.D. and Feher, G. Ann. Rev. Phys. Chem. 1996, 47, 171.

S5. Durbin, S.D. and Feher, G. J. Crys. Growth 1986, 76, 3. 